\title{
Electrical properties of ion irradiated polypropylene films
}

\author{
N L SINGH*, ANITA SHARMA, V SHRINET ${ }^{\dagger}$, A K RAKSHIT ${ }^{\S}$ and D K AVASTHI \\ Department of Physics, ${ }^{\S}$ Department of Chemistry, M.S. University of Baroda, Vadodara 390 002, India \\ ${ }^{\dagger}$ Electrical Research and Development Association, Makarpura, Vadodara 390 010, India \\ ${ }^{\ddagger}$ Nuclear Science Centre, Aruna Asaf Ali Marg, New Delhi 110 067, India
}

MS received 1 February 2003; revised 19 January 2004

\begin{abstract}
The effect of high-energy $(50 \mathrm{MeV}) \mathrm{Li}^{3+}$ ion beam irradiation on polypropylene (PP) film has been studied in the fluence range $2.4 \times 10^{12}-1.5 \times 10^{14}$ ions $/ \mathrm{cm}^{2}$. The a.c. electrical properties of PP films were measured in the frequency range from $0.05-100 \mathrm{kHz}$, and at temperature range between 30 and $140{ }^{\circ} \mathrm{C}$. This study indicates two peaks at $60^{\circ} \mathrm{C}$ and $120^{\circ} \mathrm{C}$ with comparatively high magnitudes. There is an exponential increase in conductivity with $\log$ of frequency and the effect is significant at higher fluences. The loss factor $(\tan \delta$ ) vs frequency plot suggests that PP film based capacitors may be useful below $10 \mathrm{kHz}$. The capacitance is constant over a wide temperature range up to $130^{\circ} \mathrm{C}$. FTIR spectra of the PP films before and after irradiation indicate that intensity of $\mathrm{C}-\mathrm{H}$ stretching vibration at $2900 \mathrm{~cm}^{-1}$ is modified. The presence of many new peaks with the increase of fluence suggests the formation of alkanes and alkynes which might be responsible for the observed changes in the dielectric and electrical properties of PP films.
\end{abstract}

Keywords. Ion irradiation; conductivity; dielectric loss; capacitance; polypropylene.

\section{Introduction}

A major goal of research on conducting polymers is to create materials, which not only have conductivities comparable to metals or semiconductors, but which are also easily processed and stable at normal ambient temperature. Nevertheless, most polymeric materials are normally dielectric and many are considered to be good insulators. Polymers having conductivities above the semiconductor range have been produced by chemically doping polymers having conjugated or unsaturated backbones. The increased conductivity achieved by the addition of dopants can be unstable, and may decrease with the loss of dopant. The advantage of ion irradiation over chemical doping lies in the fact that irradiation can utilize additives that cannot be applied chemically because of their low volatility and/or insolubility in suitable solvents. Because of the thermal instability of chemically doped conducting polymers and problems with regard to their practical utilization, many experiments aimed to increase the electrical conductivity of polymers by ion irradiation have been reported and discussed (Venkatesan et al 1987; Davenas et al 1990; Marletta 1990; Srivastava and Virk 2000). Interactions between electromagnetic radiation e.g. electrons, gamma rays, $\mathrm{X}$-rays etc and polymer chains are different than ions because of the high value of energy loss and the spatial distribution of deposited energy (Wang et al 1991; Calcagno et al 1992; Mishra et al 2001; Ravat et al 2001).

\footnotetext{
*Author for correspondence
}

Polypropylene continues to be an important engineering polymer because of its several advantages in cost and performance. For health care and packaging applications PP has the advantage of being nontoxic and inert to liquids and drugs. PP is used as capacitor dielectric because of its very low dielectric loss and excellent dielectric strength. Polypropylene belongs to the family of polyolefin. It is a vinyl polymer having hydrogen atom substituents, $\left(-\mathrm{H}_{2} \mathrm{C}-\mathrm{CH}_{2}-\right)_{n}$ or $\left(-\mathrm{H}_{2} \mathrm{C}-\mathrm{CRH}-\right)_{n}$ and undergoes dominant homolithic rupture of $\mathrm{C}-\mathrm{H}$ bonds to form hydrogen free radicals, which cross-link with each other. With increasing fluence, the degree of cross-linking increases. Being highly crystalline, PP exhibits high stiffness, hardness and tensile strength. It has excellent mechanical and electrical properties. In the present work, the effect of $50 \mathrm{MeV} \mathrm{\textrm {Li } ^ { 3 + }}$ ion irradiation on polypropylene (PP) has been studied.

\section{Experimental}

Three pieces of PP film (density, $0.9 \mathrm{~g} / \mathrm{cm}^{3}$ ), each of size $1.5 \times 1.5 \mathrm{~cm}^{2}$, were cut from the commercial sheet received from Garware group of industries. Thickness of polymer was measured by micrometer. It was measured at different places chosen randomly and average value was found to be $320 \mu \mathrm{m}$. Three samples were mounted on a vertical vacuum shielded sliding ladder and irradiated in general purpose scattering chamber (GPSC) using $50 \mathrm{MeV}$ $\mathrm{Li}^{3+}$ ion beam available from 15UD pelletron at the $\mathrm{Nu}-$ clear Science Centre, New Delhi. The residual pressure in 
the target chamber was $10^{-6}$ Torr. Irradiation was made at three fluences ranging from $2.4 \times 10^{12}$ to $1.5 \times 10^{14}$ ions/ $\mathrm{cm}^{2}$. The beam current was maintained around $20 \mathrm{nA}$ in order to avoid excessive heating. The irradiated samples were stored at room temperature in air.

The resistance of the samples was measured by a constant voltage method. This technique results in a much more stable and reliable impedance measurement than the conventional constant current method. Electrical contact was made by applying an air drying type of silver paste. The resistance, capacitance and dielectric loss measurements were made with the help of variable frequency LCR meter (General Radio, USA: model 1689) in the frequency range $0.05-100 \mathrm{kHz}$. The measurements at different temperatures were also made using a specially designed sample holder where the temperature was measured and maintained with a controller using copper-constant thermocouple. The a.c. conductivity was calculated using the relation, $\rho=t / R A\left(\Omega^{-1} \cdot \mathrm{cm}^{-1}\right)$, where $R$ is the resistance in ohms, $A$ and $t$ are the cross-sectional area of the electrode and thickness of the sample, respectively.

The functional group analysis of irradiated PP films was carried out by means of FTIR spectroscopy (Bomen, Canada, model MB-104) in the wave number range 500$4000 \mathrm{~cm}^{-1}$ with a resolution of four wave numbers.

\section{Results and discussion}

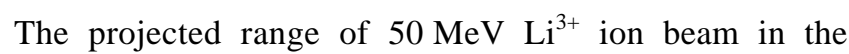
polypropylene was calculated to be $526 \mu \mathrm{m}$ using SRIM2000 code (Ziegler 2000), which was 1.64 times the thickness of PP samples. When an energetic ion passes through a material medium, it loses its energy in the medium by two main processes, viz. by interacting with target electrons and target nuclei. The energy deposition leads to production of new reactive species (radicals, gas); defects (unsaturations, scissions, crosslinks) and heat (Chailley et al 1995). The electronic stopping power of the beam $(\mathrm{d} E / \mathrm{d} x)_{e}$ is $5.358 \mathrm{eV} / \AA$ and the nuclear stopping power of the beam $(\mathrm{d} E / \mathrm{d} x)_{n}$ is $3.227 \times 10^{-3} \mathrm{eV} / \AA$. The energy deposited in the medium (PP film) comes out to be $38 \mathrm{MeV}$. The PP films were irradiated for $1 \mathrm{~min}, 10 \mathrm{~min}$ and $60 \mathrm{~min}$ durations. The corresponding fluences were $2.4 \times 10^{12}, 3.4 \times$ $10^{13}$, and $1.5 \times 10^{14}$ ions $/ \mathrm{cm}^{2}$, respectively.

\subsection{Conductivity vs frequency}

Figure 1 shows the dependence of conductivity of PP films on $\log$ frequency $(F$ in $\mathrm{Hz})$ at ambient temperature for pristine and irradiated samples. A sharp increase in conductivity has been observed at $20 \mathrm{kHz}$. It is also observed that conductivity increases as fluence increases. The increase in conductivity due to irradiation may be attributed to scissioning of polymer chains and as a result, increase of free radicals, unsaturation etc. When an a.c. field of sufficiently high frequency is applied to a metal polymer metal structure, it may cause a net polarization, which is out of phase with the field. This results in a.c. conductivity, it appears at frequency greater than that at which traps are filled or emptied (Jonscher 1977; Singh et al 2003).

\section{$3.2 \tan \delta$ vs temperature}

Figure 2 shows the behaviour of dielectric loss versus temperature at frequency, $10 \mathrm{kHz}$, for pristine and irradiated samples. The small and activated phenomenon at low temperature $\left(60^{\circ} \mathrm{C}\right)$ is due to local motion of pieces of the polymer backbone in agreement with the observations of Wintersgill and Fontanella (1989), while the large peak is due to heat distortion (or heat deflection) temperature (HDT) at $120^{\circ} \mathrm{C}$. In the case of an amorphous poly-

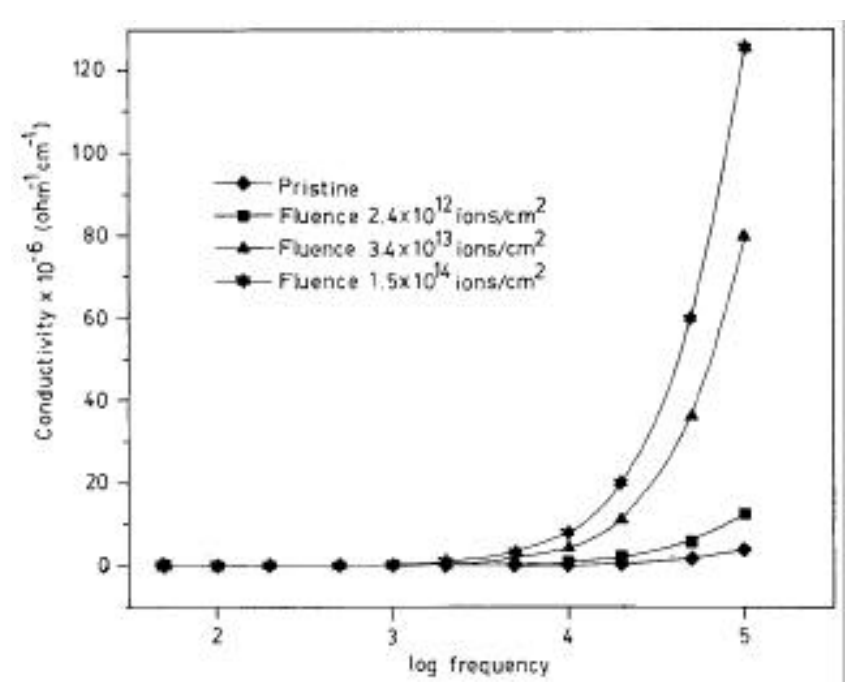

Figure 1. Plot of conductivity vs $\log$ frequency for pristine and irradiated samples at ambient temperatures.

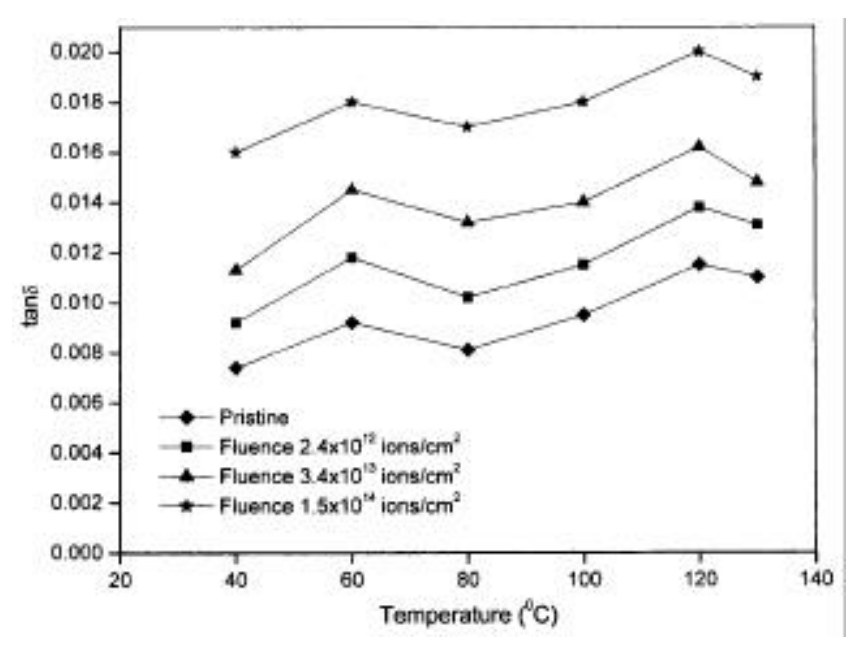

Figure 2. Plot of dissipation factor $(\tan \delta)$ vs temperature for pristine and irradiated samples at $10 \mathrm{kHz}$ frequency. 
mer, HDT is slightly $\left(10\right.$ to $\left.20^{\circ} \mathrm{C}\right)$ lower than the $T_{\mathrm{g}}$ as determined by thermal techniques, while in the case of semicrystalline polymers, HDT is more closely identified with $T_{\mathrm{m}}$ (Fried 2000). HDT is a useful indicator of the temperature limit above which polymers cannot be used for structural (load-supporting) applications. It is observed from the $\tan \delta$ vs temperature plot that maximum in dielectric loss $(\tan \delta)$ at a particular temperature is independent of the fluence. The value of $\tan \delta$ is also found to increase with increasing fluence. The dielectric loss is due to the perturbation of the phonon system by an electric field, the energy transferred to the phonons dissipated in the form of heat.

\section{$3.3 \tan \delta$ vs frequency}

Figure 3 shows the behaviour of $\tan \delta$ measured as a function of frequency at different temperatures keeping fluence constant $\left(1.5 \times 10^{14}\right.$ ions $\left./ \mathrm{cm}^{2}\right)$. It is observed that with the increase in frequency, dielectric loss ( $\tan \delta$ ) shows moderate increase up to $10 \mathrm{kHz}$ at all temperatures, suggesting that PP films can be used as dielectric in capacitors being used below $10 \mathrm{kHz}$ frequency.

Figure 4 shows the behaviour of dielectric loss with increase in frequency at different fluences at room temperature. It is observed that loss factor increases moderately as frequency increases. It is also observed that the loss factor increases as fluence increases. The growth in $\tan \delta$ and thus increase in conductivity is brought about by an increase in the conduction of residual current and the conduction of the absorption current (Tareev 1975).

\subsection{Capacitance vs frequency}

Figure 5 shows the behaviour of capacitance as a function of frequency at different fluences $\left(2.4 \times 10^{12}-1.5 \times 10^{14}\right.$ ions/

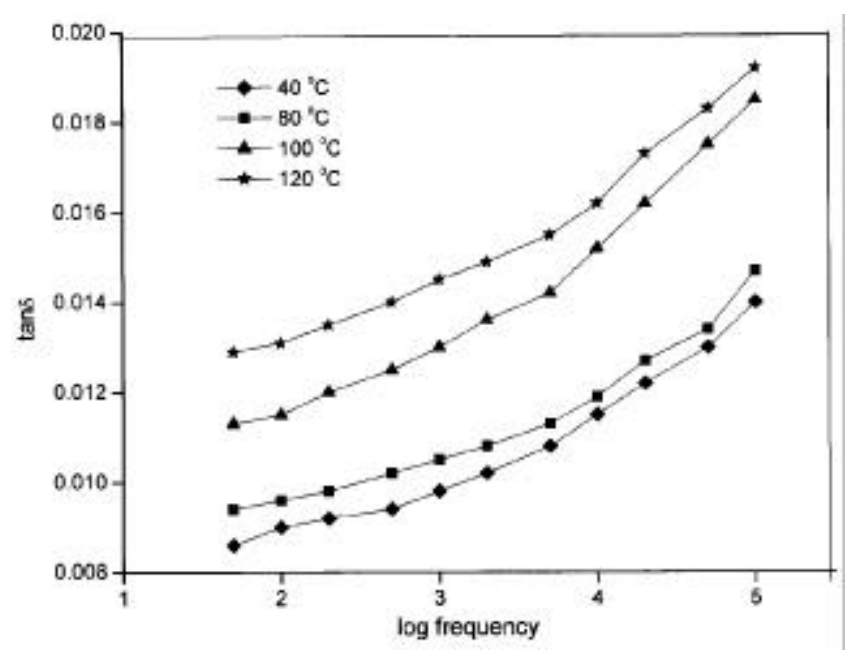

Figure 3. Plot of dissipation factor $(\tan \delta$ ) vs $\log$ frequency at different temperatures keeping fluence constant. $\mathrm{cm}^{2}$ ) for $\mathrm{Ag}-\mathrm{PP}-\mathrm{Ag}$ structure. It is observed that capacitance remains constant in the frequency range $0 \cdot 05-100 \mathrm{kHz}$. At these frequencies the motion of the free charge carrier is constant and so capacitance is constant. It is also observed that capacitance increases as ion fluence increases. The increase in capacitance may be attributed to the chain scission and as a result the increase in the number of free radicals etc. It is an interesting observation because a lot of PP based capacitors are used in nuclear plants and are bound to be exposed to nuclear radiation.

\subsection{Capacitance vs temperature}

Figure 6 shows capacitance as a function of temperature at a constant frequency of $10 \mathrm{kHz}$ for pristine and irradiated samples. Capacitance is almost independent of tem-

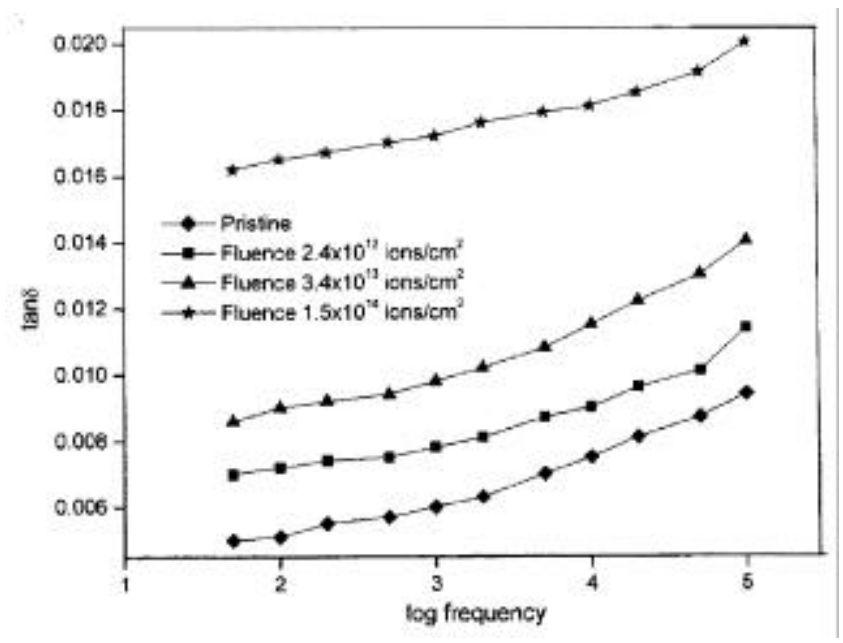

Figure 4. Plot of dissipation factor $(\tan \delta)$ vs $\log$ frequency at different fluences at ambient temperature.

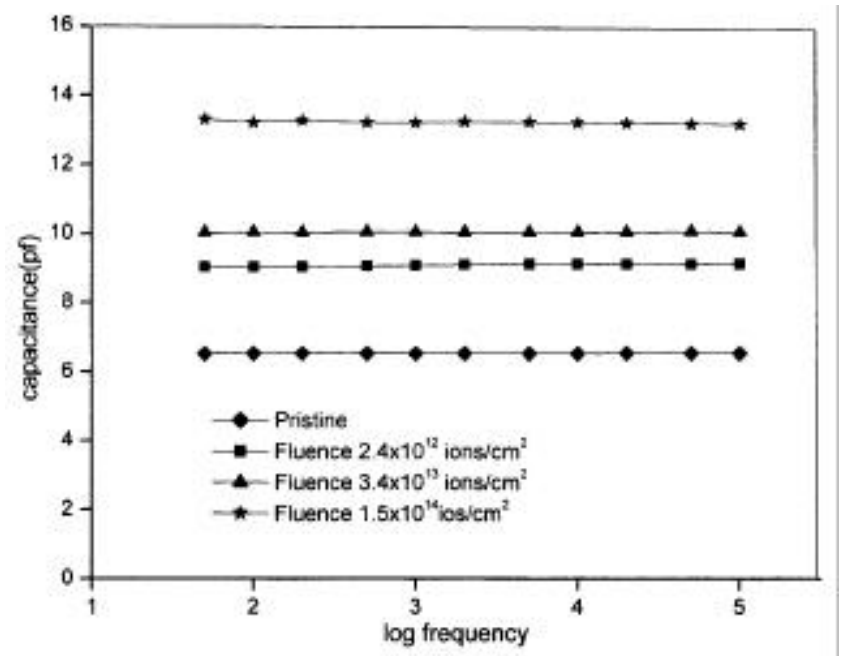

Figure 5. Plot of capacitance vs log frequency at different fluences. 
perature. The negligible change (about 6\%) in capacitance with temperature could be due to the effect of conductance corresponding to absorption current. It is also observed that capacitance increases with increase of fluence. This increase in capacitance may be attributed to the chain scission and results in an increase of free radicals etc.

\subsection{FTIR analysis}

The change in chemical structure of polypropylene due to ion irradiation is identified by FTIR spectroscopy. The IR spectra of pristine and irradiated PP films are shown in figure 7 . The most important change with higher fluence $\left(1.5 \times 10^{14}\right.$ ions $\left./ \mathrm{cm}^{2}\right)$ occurs at $\sim 2900 \mathrm{~cm}^{-1}$. This particular wave number is associated with $\mathrm{C}-\mathrm{H}$ stretching. The

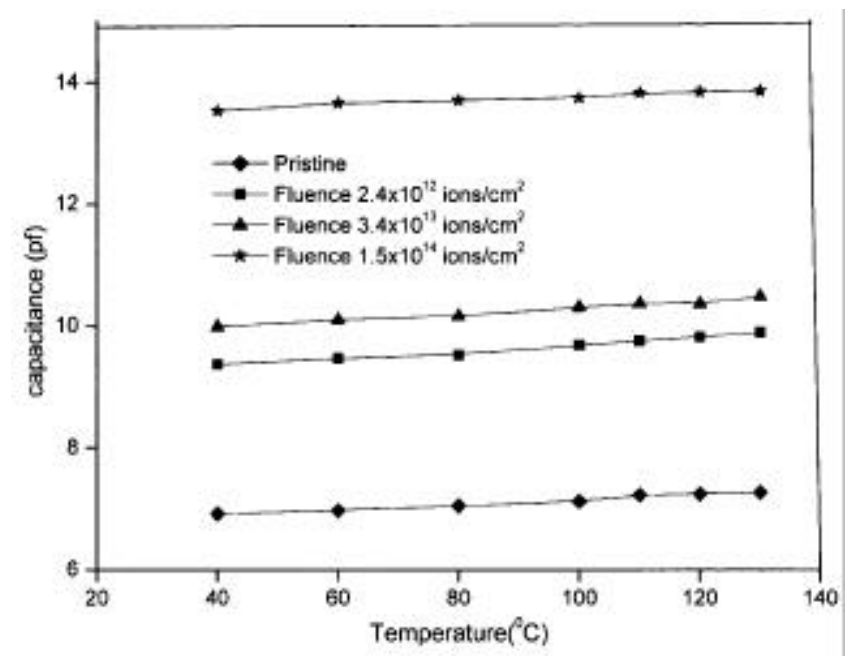

Figure 6. Plot of capacitance vs temperature at different fluences.

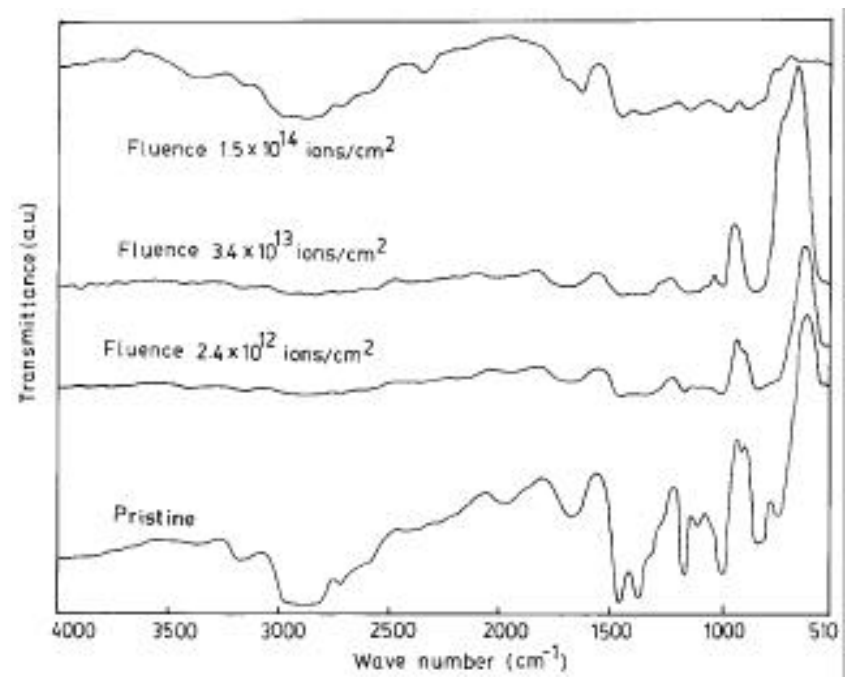

Figure 7. FTIR spectra of pristine and irradiated PP samples. decrease in intensity at this point indicates that the $\mathrm{C}-\mathrm{H}$ bond is affected to some extent probably because of breaking of some of the bonds.

The main effect of irradiation is the formation of new bonds, free radicals, double bonds etc. The PP has saturated bonding. The irradiation is expected to give rise to $\mathrm{C}=\mathrm{C}$ observed in $1680-1620 \mathrm{~cm}^{-1}$ region. It is seen that some absorbance occurs around this region. Moreover, there is some absorption at $1671 \mathrm{~cm}^{-1}$ even for pristine sample, which could be due to the presence of unsaturation. After a fluence of $3.4 \times 10^{13}$ ions $/ \mathrm{cm}^{2}$, the absorbance is observed at $1450 \mathrm{~cm}^{-1}$ which is also seen at the fluence of $2.4 \times 10^{12}$, or $1.5 \times 10^{14}$ ions $/ \mathrm{cm}^{2}$ as well as in the pristine sample. This may be due to the fact that the $\mathrm{C}-\mathrm{H}$ bonding does not get affected due to irradiation, whatever the fluence is, i.e. the nature and characteristic of $\mathrm{C}-\mathrm{H}$ bond do not change. The spectra have many absorbance over the whole range and some of them are difficult to decipher. The presence of alkene and alkyne groups in pristine sample seem to get modified by irradiation and can be noted by the change in the position and intensity of absorbance in the $\sim 630-900 \mathrm{~cm}^{-1}$ region. The irradiation also affects the polymer by forming carbonaceous materials which could also be observed by naked eyes. The IR spectra indicate the formation of such materials. Moreover, the interaction between the $\mathrm{H}$ of PP with oxygen from air may also be possible making the IR spectra reasonably complicated. Some indication of it can be found in the presence of absorbance at the $3500 \mathrm{~cm}^{-1}$ region. The $984 \mathrm{~cm}^{-1}$ band is not affected much by irradiation though $1168 \mathrm{~cm}^{-1}$ band is affected showing that $3 / 1$ helix structure gets somewhat affected by $\mathrm{Li}^{3+}$ irradiation though $\mathrm{H}^{+}$irradiation does not do so (Mishra et al 2001).

\section{Conclusions}

The ion irradiation of PP films leads to chain scission and cross linking and as a result there are changes in the dielectric properties. The value of capacitance and loss factor increases with increase of fluence. The loss factor shows a moderate increase up to $10 \mathrm{kHz}$ frequency at all temperatures suggesting that PP film capacitors may be useful below $10 \mathrm{kHz}$ frequency. The FTIR spectra shows a decrease in intensity of $2900 \mathrm{~cm}^{-1}$ peak, which is associated with stretching vibrations of $\mathrm{C}-\mathrm{H}$ bond. The presence of many new peaks with the increase of fluence suggests the formation of alkenes and alkynes which might be responsible for changing the capacitance and $\tan \delta$ values.

\section{Acknowledgements}

The authors are grateful to Prof. G K Mehta, Nuclear Science Centre, New Delhi, for support and encouragement. Financial support given by NSC, New Delhi, is also gratefully acknowledged. 


\section{References}

Calcagno L, Compagnini G and Foti G 1992 Nucl. Instrum. \& Meth. B65 413

Chailley V, Balanzat E and Dooryhee E 1995 Nucl. Instrum. \& Meth. B105 110

Davenas J, Thevenard P H, Boiteux G, Fallavier M and Xu X L 1990 Nucl. Instrum. \& Meth. B46 317

Fried Joel R 2000 Polymer science and technology (New Delhi: Prentice Hall of India Pvt Ltd) p. 155

Jonscher A K 1977 Nature 267673

Marletta G 1990 Nucl. Instrum. \& Meth. B46 295

Mishra R, Tripathy S P, Dwivedi K K, Khathing D T, Ghosh S, Muller M and Fink D 2001 Radiat. Measure. 33845

Ravat B, Grivet M, Grohens Y and Chambaudet A 2001 Radiat. Measure. 3431
Srivastava A K and Virk H S 2000 Bull. Mater. Sci. 23 533

Singh N, Sharma A and Avasthi D K 2003 Nucl. Instrum. \& Meth. B206 1120

Tareev B 1975 Physics of dielectric materials (Moscow: Mir Publication)

Venkatesan T, Calcagno L, Elman B S and Foti G 1987 Ion beam modification of insulators (eds) P Mazzoldi and G Arnold (Amsterdam: Elsevier) p. 301

Wintersgill M C and Fontanella J J 1989 Polymer electrolyte review (eds) H J R Mc Callum and C A Vincent (London: Elsevier Applied Science) Vol. 2, p. 43

Wang Y Q, Bridwill L B, Giedd R E and Marphy M J 1991 Nucl. Instrum. \& Meth. B56/57 660

Ziegler J F 2000 SRIM-2000 code, The stopping range of ions in matter (New York: IBM Research) pp 1-28 